\title{
Localization of convective breaking in a perturbed stratified atmosphere
}

\author{
C. Petulachin (*) - C. Tebalidi $(* *)$
}

Received on October 24th, 1975

\begin{abstract}
SUmarr. - The reaction to perturbations at the bottom of a stably stratified layer characterized by small periodical variations of the thermal stability parameter and capped by a, less stable, semi-infinite layer has been studied. When resonance conditions for internal zero order waves (represented by solutions obtained neglecting the small periodical variations of the stability parameter itself) trapped in the lower layer are satisfied, secondary waves, generated by the interaction of zero order waves and the small variations af the stability parameter, turn out to be resonant too. Some information ean then be obtained on the localization along the vertical of convective breaking (and turbulent patches) in the lower layer, taking into account the superposition of zero order and secondary waves. These results seem to be relevant for the comprehension of the dynamies of the atmospheric inversions as well as of the oceanic thermocline.
\end{abstract}

Riassunto. - Viene studiata la reazione, a perturbazioni applicate al fondo, di uno strato di fuido stratificato, caratterizzato da una piccola variazione periodica del parametro di stratificazione lungo la direzione verticale, sovrastato da uno strato seminfinito di fluido stratificato più debolmente. Viene messo in evidenza il fatto che, quando le condizioni di risonanza per le onde interne di ordine zero (rappresentate dalle soluzioni ottenute trascurando le piccole variazioni periodiche del parametro di stabilita) intrappolate nello strato inferiore sono soddisfatte, le onde secondarie generate dalla interazione delle onde di ordine zero e le variazioni periodiche del parametro di stabilità, risultano essere anch'esse risonanti. Da questa analisi teorica è possibile ottenere informazioni sulla localizzazione (lungo la verticale) delle instabilità convettive (strati turbolenti) nello strato più basso. Questo risultato sembra essere rilevante per la comprensione della dinamica delle inversioni atmosferiche e della termoclina oceanica.

(*) Istituto di Fisica dell'Università di Urbino.

$\left({ }^{* *}\right)$ Dipartimento di Matematica dell'Università di Ancona. 


\section{1 - INtroduction}

The reaction of a non rotating stratified atmosphere to external perturbations is most commonly analyzer assuming the constancy of the stratification parameter along the vertical direction. Layered models are, as well, considererl, the stratification parameter being constant in each layer. The effect of vertical variations of the stratification parameter on a length scale much larger than the vertical wave-number of the perturbations is also studied malking use of the W.K.B.J. approach.

Experimental data show however how the thermal stability parameter pattern is characterized by variations on many length seales along the vertical direction in the atmosphere as well as in the ocean, particularly in those zones characterized by strong mean thermal stratification (athmospheric inversion layers and oceanic thermoclines) (13).

As said above, such small variations of the stability parameters are usually ignored in the analysis of the reaction of a stratified atmosphere to external perturbations. It can be shown, however, that the role played by such small variations can become relevant, at least in some particular cases. The case analyzed in the present paper is connecter with the rynamics of perturber stable layers capped by a, less stable, semi-infinite atmosphere and, in particular, with the dynamies of wave trapped in the lower, more stable, layer and resonant to some external forcing at the bottom. An analogous problem was treated in a previous paper (11); in that paper however the eflect of small variations of the stability parameters in the lower layer, which is consilered here, was not taken into account. The pattern of the thermal stratification parameters in the stable layers conld be properly represented by a constant positive value plus a ranclom function; this case has been analyzer theoretically by different author $(2,1,5)$; and some results have been obtained suggesting the important role played by such random variations in the propagations of internal waves. $\Lambda$ s said before, we are interested in a quite different phenomenon and the assumptions that will be marle are suggested by the particular problem considered here.

Orlanski ( $\left.{ }^{7}\right)$ has widely investigated the dynamies and the breaking of resonant trapper internal waves in layers characterized by a constant thermal stratification and has suggester that convective insta- 
bility is the most probable mechanism of breaking in this case. Our analysis is fundamentally an extension of Orlanski's analysis except for some generalizations that seem to suggest certain important features in the localization of breaking zones for resonant phenomena in layers characterized by small variations of the stability parameters along the vertical direction.

\section{2 - THE THEORETICAL APPROACH}

The intrinsic difficulties of the analysis have suggested the use of a simplified two-dimensional model for an inviscid Boussinesq fluid. The whole fluid is considered at rest and the Brunt-Vaisala distribution along the vertical (see figure 1) is given by:

$$
\begin{array}{ll}
N^{2}=N_{0}^{2}(1+\varepsilon \sin \gamma z) & \text { for } 0<z<h \\
N^{2}=N_{1}^{2} & \text { for } z>h
\end{array}
$$

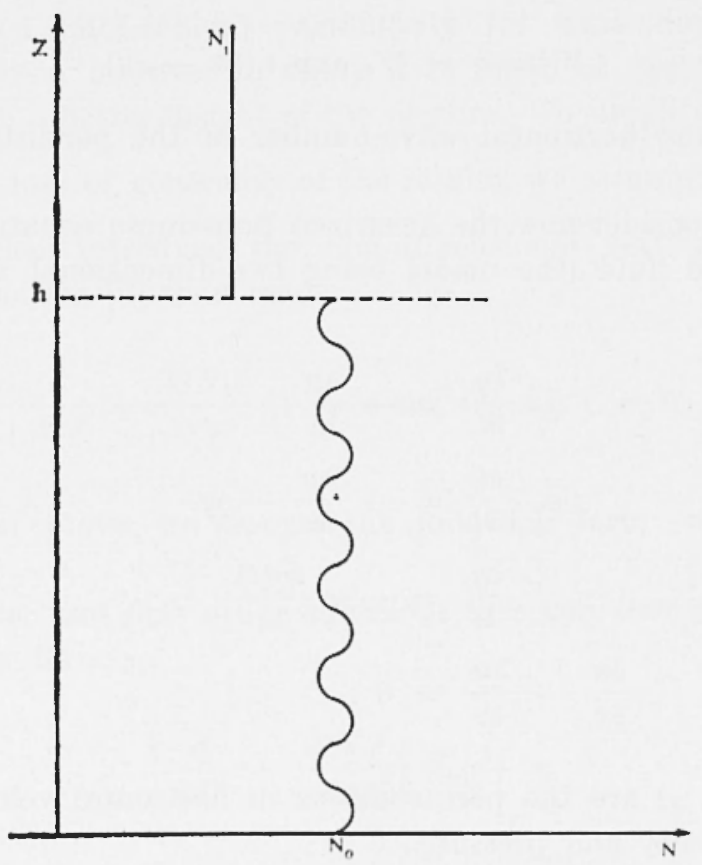

Fig. 1 - sketch of the theoretical Brunt-Vaisäla frequency distribution (the vertical scale of the oscillations of the lower layer is enlarged). 
It has to be noticed that $N^{2}$ actually shows (as stated in the previous paragraph) variations on many different length scales along the vertical direction and that the form assumed for it in $0<z<h$ means that we take into account a single length-scale only.

The following assumptions are also made:

$$
\begin{aligned}
& N_{0}>N_{1} \\
& \varepsilon \ll 1 \\
& h=n \frac{2 \pi}{\gamma}
\end{aligned}
$$

The first of assumptions [2] has been made in orler to have the possibility of studying waves trapped in the lower layer.

The second condition assures also the absence of gravitationally unstable zones in the lower layer, while the third condition has been introduced to avoid further mathematical complications in the theory, without any loss of generality of the results.

$A$ perturbation in the vertical velocity is also applied at $z=0$ in the form

$$
W / z=o=W_{0} \exp i(k x-\omega t)
$$

where $k$ is the horizontal wave-number of the perturbation and (1) its frequency.

Let us consider now the linearized Boussinesq equations in a stratified inviscid fluid (the model being two-rlimensional and the fluid at rest):

$$
\begin{aligned}
\bar{\varrho}_{0} \frac{\partial u}{\partial t} & =-\frac{\partial p}{\partial x} \\
\bar{\varrho}_{0} \frac{\partial w}{\partial t} & =-\frac{\partial p}{\partial z}-\varrho g \\
\frac{\partial o}{\partial t} & =-w \frac{\partial \varrho(z)}{\partial z} \\
\frac{\partial u}{\partial x}+\frac{\partial w}{\partial z} & =0
\end{aligned}
$$

where $(u, w, \varrho)$ are the perturbations in horizontal velocity, vertical velocity, density and pressure.

$\bar{g}_{0}$ is the average density of the whole fluid.

$\bar{Q}_{0}$ is the average value of the density in a horizontal plane. 
Equations [3] can be combined into a single differential equation for the vertical velocity; if we assume the following form for $v(x, z, t)$ :

$$
v(x, z, t)=\hat{v}(z) \exp i(k x-\omega t)
$$

equations [3] reduce to

$$
\left\{\frac{\mathrm{d}}{\mathrm{d} z^{2}}+k^{2}\left(\frac{N^{2}}{\omega^{2}}-1\right)\right\} \hat{w}(z)=0
$$

where $N^{2}=-\frac{g}{\bar{\varrho}_{0}} \frac{\mathrm{d} \bar{\varrho}}{\mathrm{d} z}$ is the squared $\mathrm{B}$. V. frequency.

By the insertion of the expression of $N^{2}$ assumed for the lower layer into equation [4], we obtain

$$
\left\{\frac{\mathrm{d}}{\mathrm{d} z^{2}}+k^{2}\left[\left(\frac{N_{\mathrm{o}}^{2}}{\omega^{2}}-1\right)+\frac{N_{0}^{2}}{\omega^{2}} \varepsilon \sin \gamma z\right]\right\} \hat{w}(z)=0
$$

Equation [5] is a typical Mathieu-Mill equation that has been widely studied (McLachlan) particularly for time dependent problem. We prefer however to study it in terms of perturbative analysis to obtain a better insight of the physics. To simplify the analysis without any loss of generality of the results, we assume $\frac{N_{0}{ }^{2}}{(1)^{2}} \gg 1$.

We further introduce the non-dimensional vertical coordinate $z^{\prime}=\gamma z$. Equation [5] then reads:

$$
\left\{\frac{\mathrm{d}}{\mathrm{d} z^{\prime 2}}+\frac{l^{2} N_{\mathrm{o}}^{2}}{\omega^{2} \gamma^{2}}\left(1+\varepsilon \sin z^{\prime}\right)\right\} \hat{\imath}\left(z^{\prime}\right)=0
$$

As stated above, we assume the following form $\hat{v}\left(z^{\prime}\right): \hat{w}_{0}\left(z^{\prime}\right)+$ $\hat{w}_{\mathrm{l}}\left(z^{\prime}\right) \varepsilon+\ldots$

Zero order and first order equations in $\varepsilon$ can then be written in the following forms:

$$
\begin{gathered}
\left\{\frac{\mathrm{d}}{\mathrm{d} z^{\prime 2}}+\frac{k^{2} N_{\mathrm{o}}^{2}}{\gamma^{2} \omega^{2}}\right\} \hat{w}_{\mathrm{o}}\left(z^{\prime}\right)=0 \\
\left\{\frac{\mathrm{d}}{\mathrm{d} z^{\prime 2}}+\frac{k^{2} N_{\mathrm{o}}^{2}}{\gamma^{2} \omega^{2}}\right\} \hat{w}_{\mathrm{l}}\left(z^{\prime}\right)=\frac{\hbar^{\prime .9} N_{\mathrm{o}}^{2}}{\gamma^{2} \omega^{2}} \hat{w}_{\mathrm{o}}\left(z^{\prime}\right) \sin z^{\prime}
\end{gathered}
$$


It can be shown that the perturbation analysis produces usefu] results as long as the following relation is satisfied $\left({ }^{10}\right)$.

$$
\varepsilon \ll\left(\gamma / \lambda_{0}\right)^{4}-8\left(\gamma / \lambda_{0}\right)^{2}
$$

where $\lambda_{0}=\frac{k: N_{0}}{\omega}$.

In the semi-infinite layer $(z>h)$ both zero and first order equations will have the same form of equation [7] with $N_{0}$ replaced by $N_{1}$.

The following boundary conditions are then applied:

$$
\begin{aligned}
& \hat{w}_{0}=w_{0} \mid \\
& \hat{w}_{1}=0
\end{aligned} \quad \text { at } z=0
$$

the continuity of $\hat{w}_{0}, \frac{\mathrm{d} \hat{w}_{0}}{\mathrm{~d} z}, \hat{w}_{1}, \frac{\mathrm{d} \hat{w}_{1}}{\mathrm{~d} z}$ at $z=h$

In the range $N_{1}<(1)<N_{0}$, the zero order solution will have the form

$$
\hat{w}_{0}\left(z^{\prime}\right)=w_{0} \cos \frac{\lambda_{0}}{\gamma} z^{\prime}+w_{0} T \sin \frac{\lambda_{3}}{\gamma} z^{\prime}
$$

in the lower layer and

$$
\hat{w}_{0}\left(z^{\prime}\right)=A_{1} \exp -\frac{\mu}{\gamma}\left(z^{\prime}-n 2 \pi\right)
$$

in the upper layer where:

$A_{1}$ is an arbitrary constant that can be evaluated by the use of boundary conditions [10]

$$
\mu=l\left(1-N_{1}^{2} / \omega^{2}\right)^{\frac{1}{2}}
$$

and $T$ is a term defined by boundary conditions at $z=\hbar$

$$
T=\frac{\frac{\lambda_{\mathrm{o}}}{\mu} \sin \frac{\lambda_{\mathrm{o}}}{\gamma} n 2 \pi-\cos \frac{\lambda_{\mathrm{o}}}{\gamma} n 2 \pi}{\frac{\lambda_{\mathrm{o}}}{\mu} \cos \frac{\lambda_{\mathrm{o}}}{\gamma} n 2 \pi+\sin \frac{\lambda_{\mathrm{o}}}{\gamma} n 2 \pi}
$$

Let us now consider the solution of the first order equation in the lower layer. 
The solution of the homogeneous part will be analogous to the zero order solution and given by:

$$
A_{2} \cos \frac{\lambda_{0}}{\gamma} z^{\prime}+B_{2} \sin \frac{\lambda_{0}}{\gamma} z^{\prime}
$$

and the full solution will be given by the expression:

$$
\begin{gathered}
\hat{w}_{1}=A_{2} \cos \frac{\lambda_{0}}{\gamma} z^{\prime}+B_{2} \sin \frac{\lambda_{0}}{\gamma} z^{\prime}+ \\
+a^{+}\left[\frac{1}{2} w_{0} \sin \left(\frac{\lambda_{0}}{\gamma}+1\right) z^{\prime}-\frac{1}{2} w_{0} \mathrm{~T} \cos \left(\frac{\lambda_{0}}{\gamma}+1\right) z^{\prime}\right]+ \\
+a^{-}\left[\frac{1}{2} w_{0} T \cos \left(\frac{\lambda_{0}}{\gamma}-1\right) z^{\prime}-\frac{1}{2} w_{0} \sin \left(\frac{\lambda_{0}}{\gamma}-1\right) z^{\prime}\right]
\end{gathered}
$$

where $A_{2}$ and $B_{2}$ are arbitrary constants, and

$$
a^{+}=\frac{\alpha^{2}}{1+2 \alpha}, \quad a^{-}=\frac{\alpha^{2}}{-1+2 \alpha}
$$

where $\alpha=\frac{\lambda_{0}}{\gamma}$.

The first order solution in the layer above will have the form:

$$
A_{3} \exp -\frac{\mu}{\gamma}\left(z^{\prime}-n 2 \pi\right)
$$

where $A_{3}$ is again an arbitrary constant.

Constants $A_{2}, B$, and $A_{3}$ can be determined by applying boundary conditions $[10]$; we obtain

$$
\begin{gathered}
A_{2}=-\frac{\frac{y}{2}}{2} T\left(a^{+}-a^{-}\right) \\
B_{2}=\left(a^{+}-a^{-}\right) \frac{1}{2} w_{0} \frac{\left(\frac{\lambda_{0}}{\mu}-T\right) \cos n 2 \pi+\left(1+\frac{1}{2} \frac{\lambda_{0}}{\mu} T\right) \sin n 2 \pi}{\sin \frac{\lambda_{0}}{\gamma} n 2 \pi+\frac{\lambda_{0}}{\mu} \cos \frac{\lambda_{0}}{\gamma} n 2 \pi}
\end{gathered}
$$


Let us indicate the fraction contained in the expression of $B_{2}$ by the simbol $S$; the full first order solution then rearls:

$$
\begin{aligned}
\hat{w}_{1}\left(z^{\prime}\right)= & -\frac{1}{2} w_{0} T\left(a^{+}-a^{-}\right) \cos \alpha z^{\prime}-\frac{1}{2} w_{0} S\left(a+-a^{-}\right) \sin \alpha z^{\prime}+ \\
& +a^{+}\left[\frac{1}{2} w_{0} \sin (a+1) z^{\prime}-\frac{1}{\bar{z}} w_{0} T \cos (a-1) z^{\prime}\right]+ \\
& +a^{-}\left[\frac{1}{2} w_{0} T \cos (\alpha-1) z^{\prime}-\frac{1}{2} w_{0} \sin (a-1) z^{\prime}\right]
\end{aligned}
$$

Comparison of expressions [17] and [13] would introduce some simplification for term $S$; the results of such comparison are however quite obvious and we prefer to pass rlirectely to the analysis of the results given by zero orler and first orler solution.

By inspection of expression [16] it is easily seen that infinite values for terms containing $\left(a^{+}-a^{-}\right)$and $a^{-}$can be predicted for $\alpha=\frac{1}{2}$; this case corresponds to the well known "parametric resonance" $\left({ }^{3}\right)$. Expression [9], together with the assumption that $\varepsilon \ll 1$ in the lower layer, implies that

$$
\frac{\lambda_{\mathrm{o}}}{\gamma}-\alpha \ll 1
$$

and the "parametric resonanee" is so avoided in our analysis.

If parametric resonance case is neglected, further inspection of expression [18] shows how the amplitudes of oscillations of wavenumbers $\alpha,(\alpha+1)$ and $(\alpha-1)$, due to the presence of term $T$ in their expression, (term $S$ for $\sin x z^{\prime}$ is more complicaterl but its role can be neglected for the moment) strongly depend on the amplitude of the component $\sin \left(\alpha z^{\prime}\right)$ of the zero order solution.

Let us consider expression [13]; it is easily seen that the argument of the trigonometric functions contained there can be properly written in the form

$$
\frac{\lambda_{\mathrm{o}}}{\gamma} n 2 \pi=\frac{h}{l_{\mathrm{o}}} 2 \pi
$$

where $l_{\mathrm{o}}$ is the wave lenght of the zero orler solution. This quantity does not actually depend on $\gamma$. Let us now consider the case in which $T$ becomes very large (two obvious cases can be studied in a very simple 
form for $\frac{\lambda_{0}}{\mu} \ll 1$ or $\frac{\lambda_{0}}{\mu} \gg 1$ ); the amplitude of term $T$ (which corresponds to the resonance of zero order (solution $w_{0} T \sin \alpha z^{\prime}$ )) implies that also the amplitude of first order solutions and, in particular, cos $(\alpha+1)$ and $\cos (\alpha-1)$, becomes large. More precisely, expression [13] can in fact be written in the following form

$$
T=\frac{\lambda_{0}}{\mu} \frac{\operatorname{tn} \frac{h}{i_{0}} 2 \pi-\frac{\mu}{\lambda_{0}}}{\frac{\lambda_{0}}{\mu}+\operatorname{tn} \frac{\hbar}{l_{0}} 2 \pi}
$$

and the following conditions must be satisfied to obtain infinite value of $T$

$$
\lambda_{0}=-\mu \operatorname{tn} \lambda_{0} h=-\mu \operatorname{tn}\left(2 \pi n \frac{\lambda_{0}}{\gamma}\right)
$$

On the contrary for

$$
\lambda_{\mathrm{o}}=\mu \operatorname{tn} \lambda_{\mathrm{o}} h=\mu \operatorname{tn}\left(2 \pi n \frac{\lambda_{\mathrm{o}}}{\gamma}\right)
$$

the corresponding value of $T$ is zero.

From the first condition it is easily seen that $n$ must be large to satisfy resonance conditions.

This is a fundamental request for the validity of our approach, in good accord with assumption [19], that assures the validity of the perturbative approach. It can then be deduced, in the limits of perturbative approach, that a small oscillation along the vertical direction of $N^{2}$ in a stable layer capped by a less stable semi infinite layer and perturbed from below will give rise, in its trapping range $\left(N_{1}^{\prime}<(1)<N_{0}\right)$, to first order waves and, in particular, that, if resonance conditions for zero order waves are satisfied, resonant zero order waves will force the growth of first order waves. Some of this last waves will have vertical wavenumbers $\left(\lambda_{0}+\gamma\right)$ and $\left(\lambda_{0}-\gamma\right)$ respectively. The final result must then be represented by a superposition of the whole wave field.

\section{3 - Numerical test}

To verify the validity of the analytical results given in the previous paragraph, a numerical test has been developed. Equation [6] has been 


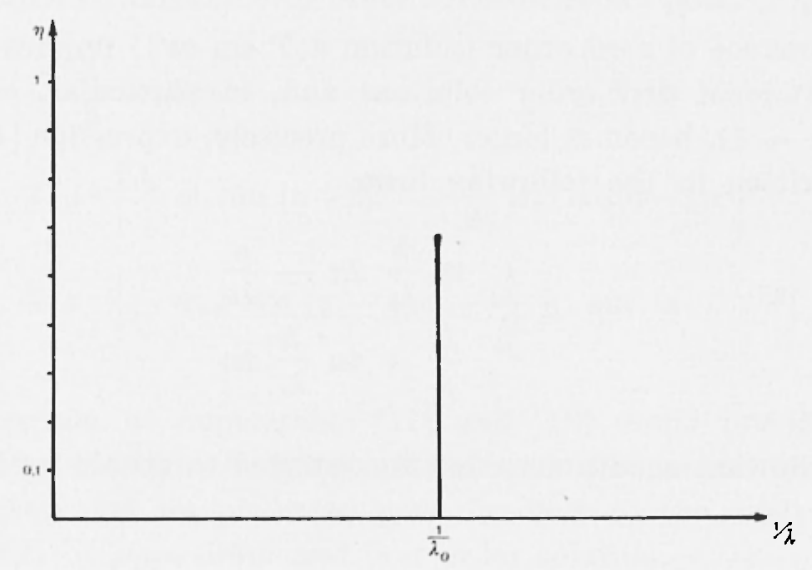

Fig. 2 - Fourior analysis of the numerical integration of equation [6] with $\varepsilon=0$

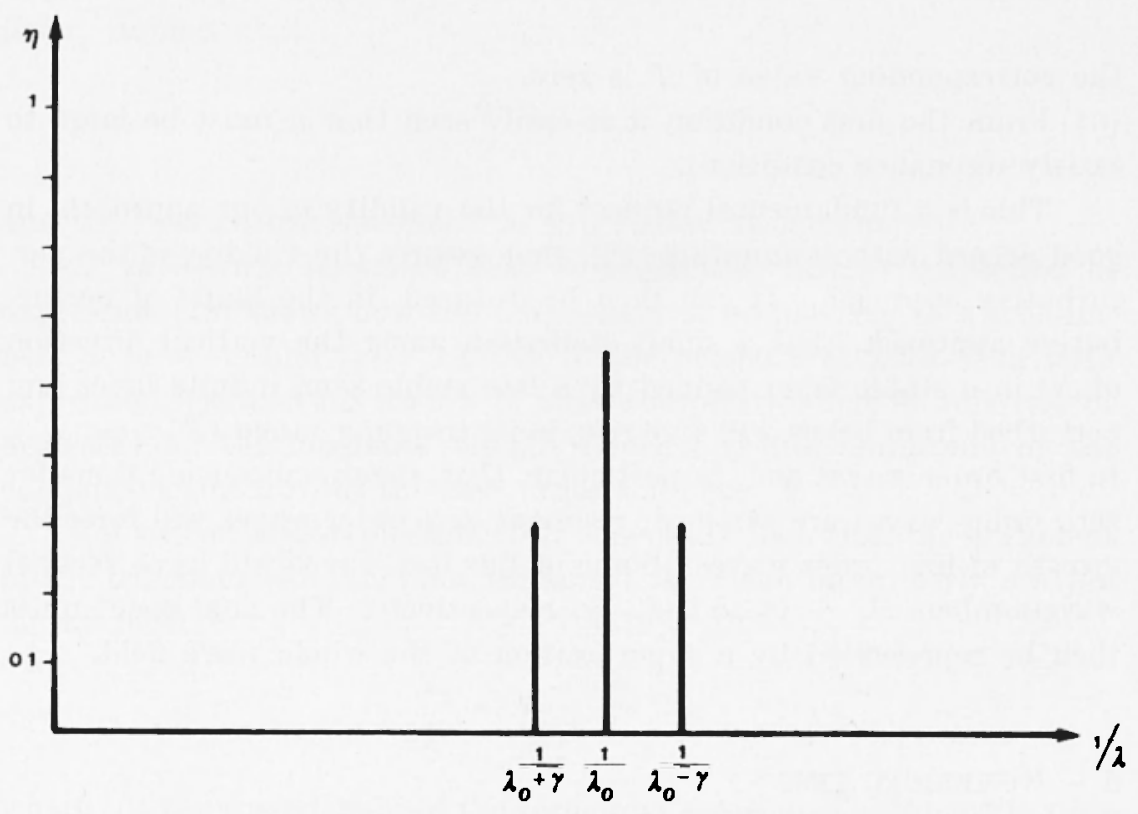

Fig. 3 - Fourier analysis of the results of numerical integration of equation [6] with $\varepsilon \cong 10^{-1}$. 
numerically integrated and Fourier analysis of the numerical solution has then been performed in conditions far from parametric resonance. Our numerical integration algorithm could not contain, unfortumately, boundary conditions but only initial value conditions.

Many experiments have however been carried on for dilferent values of the initial conditions and the results have always shown the same features in good accord with our predictions. A typical result is represented in figure 2 and 3 . In particular figure 2 , represents a typical Fourier analysis of equation [6] without the sinusoidal perturbation, while figure 3 represents the Fourier analysis with the perturbing term (of the order of $10^{-1}$ ), the other parameters being kept fixerl.

By inspection of figures 2 and 3 it is easily seen that the results of the numerical integration are quite satisfying from our point of view and can be consirlered as a partial confirm of our perturbative approach.

\section{4 - The PHysical INTERPRETATION}

A more physical interpretation of the results obtained, ean be achiever by some analysis suggresting the evolution in time of a system analogous to that considered above.

In a series of recent papers $(8,9,7)$ Orlanski has analyzed the evolution in time of resonant waves and has shown that density (as well as velocity) fluctuations connected with trapped resonant waves grow linearly in time, in the first stage of growth. He has considered a two dimensional model for an inviscid Boussinesq fuid at rest with a constant stratification $N_{0}$ and with a periodical sinusoidal forcing at the upper surface and a rigid lid at the bottom. Ciclic boundary conditions were applied along the horizontal direction $w(0)=w(D)$.

The results of such approach, based on perturbative analysis and Laplace transform method, can be expressed by the following final relation:

$$
\left|\varrho_{\mathrm{o}}\right|=\left(\frac{N_{\mathrm{o}}^{2}}{g h} \frac{\chi_{\mathrm{o}}}{D} \frac{2 k \lambda_{\mathrm{o}}}{(k / h)^{2}+\left(2 \lambda_{\mathrm{o}} / D\right)^{2}}\right) t
$$

where $\%_{0}$ is the stream function value at the top and $t$ is the time. Moreover, Orlanski has shown, both theoretically and experimentally, 
that profiles of wave fields are quite similar to those predicted by linear theory at times at which gravitational instability of resonant trapped waves starts $\left({ }^{9}\right)$. Expression [13], whose behaviour is representative of resonance condition for zero order waves, is strictly connected with the shape of zero order waves themselves as they are predicted by linear theory. The results obtained by Orlanski seem then to assure the validity of our theoretical approach, strictly connected with the behaviour of the term $T$. The results suggested, in the limits of the coarseness of the model, are that secondary waves (first order waves in our approach) ean be generated by trapped resonant internal waves and that, as a consequence, the global wave field must be considered as the superposition of primary and secondary waves. This fact introduces some new elements in the analysis of localized convective breaking of resonant trapped waves in atmospheric inversions layers and marine thermoclines $(11,8)$.

Orlanski has, in fact, quite well explained in terms of gravitational instability of resonant trapped gravity waves, in a layer characterized by a constant $N$ the generation of step-like structures in the stratification parameter. For a single wave, the criterion for the existence of convective instability can be written in the following form:

$$
u>\frac{\omega}{k}
$$

where $u$ is the horizontal wave field velocity and $\omega / k$ is the horizontal phase speed.

By our previous analysis it seems possible to suggest that, in a layer characterized by a small vertically varying structure of the stability parameter, secondary waves (with the same horizontal wave speed $\omega / k$ ) are generated and then that local constructive superposition of zero and first order waves will grenerally give rise to localized (along the vertical) turbulence generation. The horizontal velocity of the total wavefield, at levels where positive superposition will take place, will be given by the expression $\left(u_{0}+\varepsilon u_{1}\right)$ and, being the horizontal phase speed of both zero and first order waves the same, the condition for gravitational breaking will be most probably reached there. From previous analysis it could also be inferred that some of the characteristics of experimental results obtained in laboratory (4,7) could be explained in terms of the mechanism considered here assuming that our stationary stratification could be interpreted as the effect of low 
frequency, high vertical wave-number waves. An experimental test is however in planning for the proper test of the theory.

\section{5 - Coyclusions}

Our results seem fundamentally to suggest that the perturbation of a stable layer, characterized by small amplitude variations of the stability parameter along the vertical and capped by a less stable layer, will lead to a spreading of energy into different vertical wavenumbers determined by the zero order wave-number and the one of the stationary perturbation. This mechanism is particularly effective in the case of resonance of the zero configuration and seems to introduce some significant information on the localization (along the vertical) of turbulent patches due to convective instability. If the superposition of more than one scale of variations of the stability parameter along the vertical is considered, the spreading of the energy can be predicted for other vertical wavenumbers due to the linearity of our approach.

Some obvious difficulties suggest however that the more general case has to be considered from a more sophisticated point of view as non - linear interaction between secondary waves could lead to an energy cascade not predictable by our theory.

Finally, while detailed comparisons have been made with Orlansli's work our results seem to be in good accord also with those obtained for a quite similar problem for the ocean by Phillips (12).

\section{REFERENCES}

(1) Howe, MI. S., 1971. - Wave propagation in random media. "J. Fluid Mech.", 45, pp. 769-783.

(2) Keller, J. B. and Veronis, G., 1969. - Rossby waves in the presence of random currents. "J. Geophys. Res", 74, pp. 1941-1951.

(3) LaNDAU, L. and IJIFCnTIz, E., 1966. - Mécanique. "MIIR".

(4) Mcliwax, A. D. and Ronisson, R. M., 1975. - Parametric instability of internal gravity wates. "J. Fluid Mech", 67, pp. 667-687.

(5) McGoryax, R. E. and IISAK, L.A., 1973. - Internal waves is a randomly stratified fluid. "Geoplyys. Fluid Dynamies", 4, pp. 243-266.

$\left.{ }^{6}\right)$ McLacilas, N. W., 1947. - Theory and application of Mathieu Functions. Claredon Press. 
(') OrLaxsk, I., 1972. - On the breaking of standing internal gravity wares. "J. Fluid. Mech.", 54, 4, pp. 577-598.

(") ORLANski, I. and BRYAN, K., 1969. - Formation of the thermoctine step structure by large-amplitude internal grarity waves. "J. Geophys. Res.", 74. pu. $6975-6983$.

(") ORLAxski, I. and Ross, B.. 1973. - Numerical simulation of the generation of breaking internal gravity waves. "I. Geophys. Res.", 78, pp. 8808-8826.

(10) Plinson, C. E., 1974. - IIandbook of applied mathematies. Van Nostrand Reinhold Company.

(11) Piglacani C. and Juprin, R., 1975. - Resonamt trapped gravily waves and turbulenl patches in an imersion layer. "Boundary Layer Met", in print.

(12) Phillis, O. M., Gisonge, W. K., Mren, R. P., 1968. - -1 note on the interaction between internal gracily waces and currents. "Deep-Sea Res", 15, pl. $267-273$.

${ }^{(13)}$ TURnwe, J. S.. 1973. - Buoyancy elfects in /luids. "Cambridge University Press". 